\title{
On Optimization of Sensor Selection for Aircraft Gas Turbine
}

\section{Engines}

\author{
Ramgopal Mushini \\ Cleveland State University, mramgopal@gmail.com \\ Daniel J. Simon \\ Cleveland State University, d.j.simon@csuohio.edu
}

Follow this and additional works at: https://engagedscholarship.csuohio.edu/enece_facpub

Part of the Electrical and Computer Engineering Commons

How does access to this work benefit you? Let us know!

\section{Publisher's Statement}

(C) 2005 IEEE. Personal use of this material is permitted. Permission from IEEE must be obtained for all other users, including reprinting/ republishing this material for advertising or promotional purposes, creating new collective works for resale or redistribution to servers or lists, or reuse of any copyrighted components of this work in other works.

\section{Original Citation}

R. Mushini and D. Simon. (2005). On Optimization of Sensor Selection for Aircraft Gas Turbine Engines. International Conference on Systems Engineering, 9-14, doi: 10.1109/ICSENG.2005.61.

\section{Repository Citation}

Mushini, Ramgopal and Simon, Daniel J., "On Optimization of Sensor Selection for Aircraft Gas Turbine Engines" (2005). Electrical Engineering \& Computer Science Faculty Publications. 182.

https://engagedscholarship.csuohio.edu/enece_facpub/182

This Conference Proceeding is brought to you for free and open access by the Electrical Engineering \& Computer Science Department at EngagedScholarship@CSU. It has been accepted for inclusion in Electrical Engineering \& Computer Science Faculty Publications by an authorized administrator of EngagedScholarship@CSU. For more information, please contact library.es@csuohio.edu. 


\title{
On optimization of sensor selection for aircraft gas turbine engines
}

\author{
Ramgopal Mushini \\ Cleveland State University \\ Department of Electrical Engineering \\ mramgopal@gmail.com
}

\author{
Dan Simon \\ Cleveland State University \\ Department of Electrical Engineering \\ d.j.simon@csuohio.edu
}

\begin{abstract}
Many science and management problems can be formulated as global optimization problems. Conventional optimization methods that make use of derivatives and gradients are not, in general, able to locate or identify the global optimum. Sometimes these problems can be solved using exact methods like brute force. Unfortunately these methods become computationally intractable because of multidimensional search spaces. Hence the application of heuristics for a class of problems that incorporates knowledge about the problem helps solve optimization problems. Optimization of sensor selection using heuristics can lead to significant improvements in the controllability and observability of a dynamic system. The aim of this research is to investigate optimal or alternate measurement sets for the problem of aircraft gas turbine engine health parameter estimation. The performance metric is defined as a function of the steady state error covariance and the cost of the selected sensors
\end{abstract}

\section{Introduction}

A physical dynamical system model is not perfect, especially one that has been linearized, but the accuracy within a neighborhood of the operating point may be acceptable. Also, sensor measurements are limited in their accuracy because of noise and resolution. Being able to quantify these limitations is a key to achieving good estimation. A technique that is commonly used to model system degradation while still representing the system as time-invariant is to introduce biases into the linearized model. These biases model changes in the system state and output values due to system degradation. These biases can be appended to the system's state vector in which case they are sometimes called extended parameters [1]. In general, measurements change when the parameters associated with them change. In this research we attempt to determine the best measurement sets to minimize a combination of overall parameter uncertainty and cost using steady state estimation error covariance analysis. Section 2 discusses work done in the past by other authors, and Section 3 discusses the aircraft turbofan engine, its states, control inputs, health parameters and sensors. Section 4 gives an overview of sensor selection and Section 5 talks about the search algorithms and optimization using genetic algorithms. Section 6 discusses results, conclusions, and future work .

\section{Literature Survey}

Various algorithms have been proposed for sensor selection optimization. A search over all possible sensor combinations grows exponentially with the number of sensors; in our case we have a total of 22 sensors where each of 11 sensors can be used twice. So a search over 177146

This work was partially supported by the NASA Aviation Safety and Security Program at the NASA Glenn Research Center. 
combinations (a brute force technique) will be unacceptable and a more efficient method is needed. The Eigenvalue/Minimum Sensors algorithm [3] results in a sensor combination with the fewest number of sensors that produce eigenvalues in the error covariance matrix is the best sensor combination. In the Matrix Norm Algorithm, a sensor selection algorithm is used to minimize the norm of the inverse of the error covariance for the sensor combination whose inverse covariance can be calculated. Then the algorithm checks if the norm of the covariance is within a predefined boundary, thus selecting the combination that uses fewest sensors while keeping the covariance within the boundary [2]. In another approach the concept of randomization and super heuristics is used to develop a computationally efficient model for generating an optimal sensor set [3].

\section{Aircraft gas turbine engines}

An aircraft gas turbine engine simulation is used in order to obtain a linear dynamic model of the system. The health parameters that we try to estimate can be modeled as slowly varying biases. Model biases can be estimated by augmenting them to the system dynamic model [1]. A linearized model of a turbofan is obtained from MAPSS, the Modular Aero Propulsion System Simulation, a public domain aircraft gas turbine engine simulation developed by the NASA Glenn Research Center [4] .The turbofan engine modeled as MAPSS has a set of states, control inputs and outputs that describe the engine's behavior. This aircraft gas turbine engine system has 3 states, 10 health parameters and 11 sensors. The states, control inputs and sensor outputs of the MAPSS model are given below in Table 1. The parameters that have a major effect on the health of the turbofan engine are called the health parameters. Estimating these health parameters over a period of time is the objective of this research. The ten health parameters of the MAPSS model are in Table 1.

Table 1. MAPSS sensor outputs, states, control inputs, health parameters

\begin{tabular}{|c|c|c|}
\hline Sensor outputs & Health parameters & Control inputs \\
\hline 1) LPT exit pressure & 1) Fan airflow & 1) Main burner fuel flow \\
\hline 2) LPT exit temperature & 2) Fan efficiency & 2 ) Variable nozzle area \\
\hline 3) Fan exit pressure & 3) Booster tip airflow & 3 )Rear BP door variable area \\
\hline $\begin{array}{l}\text { 4) Percent low pressure spool rotor } \\
\text { speed }\end{array}$ & 4) Booster tip efficiency & \\
\hline 5) Booster inlet pressure & 5) Booster hub airflow & \\
\hline 6) HPC inlet temperature & 6) Booster hub efficiency & \\
\hline 7) HPC exit pressure & 7) High pressure turbine airflow & \\
\hline 8) HPC exit temperature & 8) High pressure turbine efficiency & States \\
\hline 9) Core rotor speed & 9) Low pressure turbine airflow & 1 )High pressure rotor speed (xnh) \\
\hline 10) Bypass duct pressure & $\begin{array}{l}\text { 10) Low pressure turbine } \\
\text { efficiency }\end{array}$ & 2) Low pressure rotor speed (xnl) \\
\hline 11) LPT blade temperature & & 3) Heat soak temperature (tmpc) \\
\hline
\end{tabular}




\section{Sensor Selection}

For systems with time invariant extended parameters appended to the state vector the minimum number of outputs required for observability is equal to the number of extended parameters [1]. A tradeoff should be made between the number of sensors and the financial cost. We could simply use all sensors to obtain the best possible health estimation. But if we can save a lot of money or effort at a very small decrease in estimation accuracy, then we may want to use fewer sensors. The goal is to a find a measure of the quality of the estimation as a function of the sensor set used. This is accomplished by applying combinations of sensors to minimize a cost function generated using the covariance of each state estimate. The timeinvariant system equations can be summarized as follows:

$$
\begin{aligned}
& \dot{x}=f(x, u, p)+w(t) \\
& y=g(x, u, p)+e(t)
\end{aligned}
$$

Here $x$ is a system state vector with 3 states, $u$ is the input vector, $p$ is the 10 -element vector of health parameters, and $y$ is the 11-element vector of measurements. The noise term $w(t)$ represents inaccuracy in the model, and $e(t)$ represents measurement noise. The system model built assumes that the health information is contained in the state variables. The goal is to find a measure of the quality of estimation as a function of the sensor set. A tradeoff should be made between sensors, cost, weight, accuracy and reliability. So, additional sensors to allow better estimates modify the given system. The concept of using multiple sensors for a single measurement produces smaller variance than that produced using a single sensor alone [1]. In order to estimate the health parameters, the original 3 states are augmented with the 10 health parameters, so the $A$ matrix is $13 \times 13$. The measurement matrix with 11 rows (corresponding to 11 sensors) can be duplicated with the same set of 11 sensors in case each sensor is used twice. This results in a measurement matrix $C$ with 11 columns (one for each state) and up to 22 rows (one for each sensor). A change in $C$ also effects the measurement noise covariance $R$, which is constructed to be consistent with $C$. These system matrices are used to produce an error covariance $P$, which will be designated as $P_{r e f}$ if all 22 sensors are used.

Consider a linear stochastic system represented by

$$
\begin{aligned}
& x_{k+1}=A x_{k}+B_{u} u_{k}+B_{w} w_{k} \\
& y_{k}=C x_{k}+v_{k}
\end{aligned}
$$

Here $x$ is the system state vector, $y$ is the measurement vector, $u$ is the input vector, $w$ is the process noise vector and $v$ is the measurement noise vector. $A, B_{u}, B_{w}$ and $C$ are matrices of appropriate dimensions. $w$ and $v$ in this case are assumed to be mutually independent and zero mean white noise. The covariances of $w$ and $v$ are given as

$$
\begin{aligned}
& E\left[w_{k} w_{k}^{T}\right]=Q \\
& E\left[v_{k} v_{k}^{T}\right]=R
\end{aligned}
$$

The steady state error covariance $P$ becomes the solution to the following equation:

$$
P=(A-A K C) P(A-A K C)^{T}+B_{w} Q B_{w}{ }^{T}+(A K) R(A K)^{T}
$$

where $K$ is the Kalman gain for the given sensor set. This is the error covariance when Kalman filtering is used for state estimation. The error variances are divided into the first 3 elements, representing the original state estimation error variances, and the last ten, representing the health parameter estimation variances. As we will be interested only in the health parameter error covariance we introduce a weighting function, which only considers the 4th through 11th 
elements of the augmented state. In order to compare the quality of estimation the following metric is defined.

$$
\begin{gathered}
J=\sum_{i=1}^{n} w_{i}\left(\sqrt{\frac{P_{i, i}(k \mid k)}{P_{i, i}^{r e f}(k \mid k)}}\right)+\frac{\text { financial cost }}{\text { ref financial cost }} \\
\text { where } \mathcal{W}_{i}= \begin{cases}0 & i=1, \ldots \ldots . .3 \\
1 & i=4, \ldots \ldots . .13\end{cases}
\end{gathered}
$$

In general, the weights $w_{i}$ could take on any values depending on the relative importance of estimating various health parameters. The goal is to minimize the metric by minimizing a combination of estimation error and financial cost. The estimation error portion of the metric is 1.0 for the reference measurement set where we use all 11 sensors twice. An approach of random generation of sensor sets will be carried out which determines the $C$ and the $R$ matrices. Now using the steady state error covariance we calculate $P$ for every new sensor set. This approach sometimes leads to an unobservable system, in which case that particular sensor set is excluded from consideration. $P_{i, i}$ represents the variance of the estimation error of the i-th state, which is the $\mathrm{i}$-th element along the main diagonal of $P$.

\section{Search Algorithms}

\subsection{Random search algorithm}

Initially a random search algorithm generates sensor sets and evaluates the cost function as shown in Equation (5). This random search algorithm is executed several times capturing the data for each execution. The probability of each sensor being in the top $x \%$ cost of sensor sets is evaluated, where $x$ is a user-specified threshold. Now, based on the probability of each sensor, a probabilistic algorithm, which generates sensor sets per their probability, is executed. The probabilistic approach improves the cost value when compared to random search by generating sensor sets with low cost. But this approach cannot ensure that the obtained solution has the least cost sensor set. So a genetic algorithm was implemented to obtain the least cost sensor set, which is validated using results of a brute force technique.

\subsection{Genetic algorithm for search optimization}

A genetic algorithm, with sensor numbers coded into genetic strings called chromosomes, is implemented. Each of these chromosomes has an associated fitness value, which is determined by the objective function in Equation (5) to be minimized. Each chromosome contains substrings called genes, which in this problem are the sensors, which contribute to fitness of the chromosome. The genetic algorithm (GA) proceeds by taking the population, which is comprised of different chromosomes, each of which is a set of sensors with fitness evaluated for each chromosome. In each successive generation the highest fit chromosomes survive and this increases the average fitness. When the GA is being used in the context of function optimization, success is measured by the discovery of strings that represent values yielding an optimum (or near optimum) of the given function [5]. The sensor selection optimization problem using GA can be expressed as the minimization of Equation (5) and with a constraint that the number of sensors for each chromosome is 11 sensors.

Genetic algorithms start with an initial population of individuals. Each individual in the initial population should meet the solution constraint. The population is arbitrarily initialized within specified bounds. Randomized processes of selection, crossover, and mutation help the population evolve towards better and better regions in the search space. The GA parameters in this study, determined by manual tuning, are given as follows. 
Initial population size $=100$

Population size $=50$

Crossover Probability $=0.9$

Mutation Probability $=0.003$ per sensor

Maximum Generations $=15$

Number of Sensors $=11$

\section{Results and Conclusions}

The brute force approach of searching all 177146 combinations of sensors could be too computationally expensive. So the search space was narrowed down by selecting only sets with no more than one repetition per sensor. This results in 25653 distinct sensor sets. The results of the brute force helped prove how good the algorithms searched the entire search space. Finding all possible sets having 11 sensors with no more than one duplicate was a tedious task. So a randomization technique to generate the optimal sensor set was implemented which reduced the computational complexity. This method proceeds by randomly generating a small number of sensor sets, computing their metric, obtaining the probability of each sensor being in a good sensor set, and then using those probabilities to generate a sensor set with minimum cost. Comparing the results of the probabilistic approach with brute force, we saw that the least cost obtained using the former method is 2.2028 , compared to the least cost of 2.1687 that was obtained using brute force. So a GA was developed by coding the sensor sets into a chromosome. The results of the GA for sensor selection were approximately the same as that of brute force. Relative optimization of cost with different methods can be summarized in Table 2 .

Table 2. Relative cost optimization

\begin{tabular}{|c|c|c|c|c|}
\hline Method & $\begin{array}{c}\text { Sensor Set } \\
\text { Evaluations }\end{array}$ & $\begin{array}{c}\text { Computation } \\
\text { (Minutes) }\end{array}$ & Best Cost & Sensor set \\
\hline Exhaustive Search & 25653 & 78 & 2.1687 & $1,2,4,5,5,6,6,7,7,8,10$ \\
\hline $\begin{array}{c}\text { Probabilistic } \\
\text { Search }\end{array}$ & 10000 & 30 & 2.2028 & $1,2,4,5,6,7,8,8,9,10,11$ \\
\hline \begin{tabular}{c} 
Genetic Algorithm \\
\hline
\end{tabular} & 850 & 3 & 2.1687 & $1,2,4,5,5,6,6,7,7,8,10$ \\
\hline
\end{tabular}

The GA optimized search eliminated sensors 3, 9 and 11 from the optimal sensor set. A histogram in Figure 1 shows the top 2000 sensor sets with same sensor cost for each sensor. The nominal sensor set having sensors 1 through 11 has a cost of 2.2693.

Future work will involve the use of probability theory to obtain the confidence that the final sensor set that is selected using probabilistic search is within some percentage of the absolute best sensor set that is available. For future work, joint probabilities can be obtained and used in the directed search in the same way that single probabilities have been used thus far particle swarm optimization [7] is a recently proposed algorithm by James Kennedy and R. C. Eberhart in 1995, motivated by social behavior of organisms such as bird flocking and fish schooling. 
PSO as an optimization tool provides a population-based search procedure in which individuals called particles change their position (state) with time. In a PSO system, particles fly around in a multidimensional search space. During flight, each particle adjusts its position according to its own experience, and according to the experience of a neighboring particle, making use of the best position encountered by itself and its neighbor. Thus, as in modern GAs, a PSO system combines local search methods with global search methods, attempting to balance exploration and exploitation. So implementation of evolutionary algorithms like particle swarm optimization is another idea to solve the sensor selection problem.

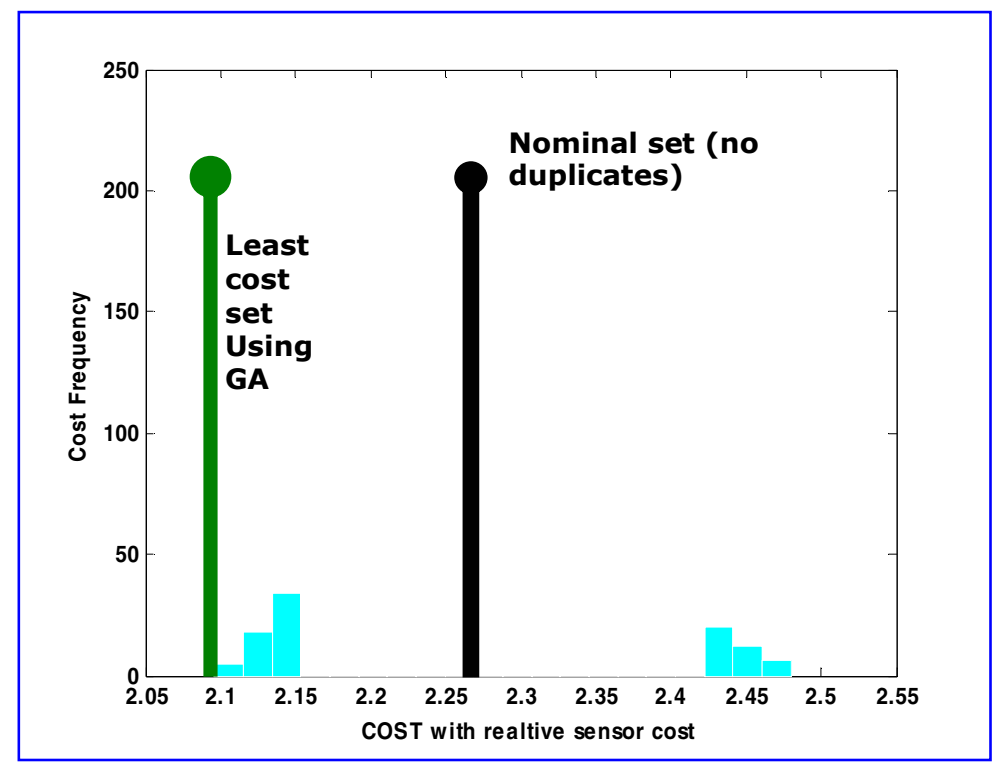

Figure 1. Histogram of the distribution of the top 2000 sensor sets

\section{References}

[1] Jonathan Litt, Sensor placement for aircraft propulsion system health management, NASA Glenn Research Center. (Unpublished Report)

[2] Lucy Y. Pao, Michael Kalandros, John Thomas, Controlling Target Estimate Covariance in Centralized Multisensor Systems, American Control Conference, Philadelphia, pp 2749-2753, June 1998.

[3] Michael Kalandros, Lucy Y. Pao, Yu-Chi Ho, Randomization and Super-Heuristics in choosing Sensor Sets for Target Tracking Applications, Proceeding of the IEEE Conference on Decision and Control, Phoenix, AZ, December 1999

[4] Khary I. Parker and Kevin J. Melcher, "Modular Aero Propulsion System Simulation (MAPSS) User's Guide," NASA/TM 2004-212968

[5] Kiego Watanabe, M.M.A.Hashem "Evolutionary Computations - New Algorithms and their Applications to Evolutionary Robots," Series: Studies in Fuzziness and Soft Computing, Vol. 147, Springer, 2004.

[6] Brent J.Brunell, Daniel E.Viassolo, Ravi Prasanth, "Model Adaptation and Nonlinear Model Predictive Control of an Aircraft Engine," ASME Turbo Expo, Vienna, 2004.

[7] James Kennedy, Russell C. Eberhart, with Yuhui Shi, Swarm Intelligence, Morgan Kaufmann, 2001

[8] Ramgopal Mushini, On optimization of sensor selection for aircraft gas turbine engines, Masters Thesis, Department of Electrical and Computer Engineering, Cleveland State University, 2004. 